\title{
c-Jun N-Terminal Kinase (JNK)-Interacting Protein-1b/Islet-Brain-1 Scaffolds Alzheimer's Amyloid Precursor Protein with JNK
}

\author{
Shuji Matsuda, ${ }^{1}$ Takashi Yasukawa, ${ }^{1}$ Yasuko Homma, ${ }^{1}$ Yuko Ito, ${ }^{1}$ Takako Niikura, ${ }^{1}$ Takako Hiraki, ${ }^{1}$ \\ Shuichi Hirai, ${ }^{2}$ Shigeo Ohno, ${ }^{2}$ Yoshiko Kita, ${ }^{1}$ Masaoki Kawasumi, ${ }^{1}$ Keisuke Kouyama, ${ }^{1}$ Tokuo Yamamoto, ${ }^{3}$ \\ John M. Kyriakis, ${ }^{4}$ and Ikuo Nishimoto ${ }^{1}$ \\ ${ }^{1}$ Department of Pharmacology and Neurosciences, KEIO University School of Medicine, Shinjuku-ku, Tokyo 160-8582, \\ Japan, ${ }^{2}$ Department of Molecular Biology, Yokohama City University School of Medicine, Kanazawa-ku, Yokohama 236- \\ 0004, Japan, ${ }^{3} T o h o k u$ University Gene Research Center, Aoba-ku, Sendai 981-8555, Japan, and ${ }^{4}$ Diabetes Research \\ Laboratory, Massachusetts General Hospital-East, Charlestown, Massachusetts 02129
}

Using a yeast two-hybrid method, we searched for amyloid precursor protein (APP)-interacting molecules by screening mouse and human brain libraries. In addition to known interacting proteins containing a phosphotyrosine-interactiondomain (PID)—Fe65, Fe65L, Fe65L2, X11, and mDab1, we identified, as a novel APP-interacting molecule, a PIDcontaining isoform of mouse JNK-interacting protein-1 (JIP-1b) and its human homolog IB1, the established scaffold proteins for JNK. The APP amino acids $\mathrm{Tyr}^{682}{ }^{6{ }^{6}} \mathrm{Asn}^{684}$, and $\mathrm{Tyr}^{687}$ in the $\mathrm{G}^{681}$ YENPTY ${ }^{687}$ region were all essential for APP/JIP-1b interaction, but neither $\mathrm{Tyr}^{653}$ nor $\mathrm{Thr}^{668}$ was necessary. APPinteracting ability was specific for this additional isoform containing PID and was shared by both human and mouse homologs. JIP-1b expressed by mammalian cells was efficiently precipitated by the cytoplasmic domain of APP in the extreme Gly ${ }^{681}$-Asn ${ }^{695}$ domain-dependent manner. Reciprocally, both full-length wild-type and familial Alzheimer's disease mutant APPs were precipitated by PID-containing JIP constructs. Antibodies raised against the $\mathrm{N}$ and $\mathrm{C}$ termini of JIP-1b coprecipitated JIP-1b and wild-type or mutant APP in nonneuronal and neuronal cells. Moreover, human JNK1 $\beta 1$ formed a complex with APP in a JIP-1b-dependent manner. Confocal microscopic examination demonstrated that APP and JIP-1b share similar subcellular localization in transfected cells. These data indicate that JIP-1b/IB1 scaffolds APP with JNK, providing a novel insight into the role of the JNK scaffold protein as an interface of APP with intracellular functional molecules.

Key words: JIP-1b/IB1; amyloid precursor protein; phosphotyrosine-interaction-domain; scaffolding protein; c-Jun $\mathrm{N}$-terminal kinase
Alzheimer's disease (AD) is the most prevalent neurodegenerative disease pathologically characterized by senile plaques in the brain. The major constituent of the plaques is $\mathrm{A} \beta$, cleaved off from the transmembrane precursor, termed amyloid precursor protein (APP). Genetic studies of familial AD (FAD) (Hardy, 1992) demonstrated that structural alterations in APP cause AD, based on the finding that certain FAD patients carry V642I/F/G mutations or an NL mutation (K595N/M596L) in $\mathrm{APP}_{695}$, a 695 residue version (Kang et al., 1987). However, how these mutations cause pathophysiology in AD and how wild-type APP (wt APP) contributes to normal functions have been little understood. Yet multiple studies (Qiu et al., 1995; Coulson et al., 1997; Gillian et al., 1997; Perez et al., 1997) have shown that wt APP performs physiological functions on the surface of neurons relevant to neurite outgrowth, neuronal adhesion, and axonogenesis, aside from its pathological role as an $\mathrm{A} \beta$ precursor. Rohn et al. (2000) and Sudo et al. (2000) independently found that anti-APP anti-

Received May 7, 2001; revised June 11, 2001; accepted June 13, 2001.

This work was supported in part by grants from the Ono Medical Research Foundation, the Ministry of Education, Culture, Sports, Science, and Technology of Japan, and the Organization for Pharmaceutical Safety and Research (OPSR). We especially thank Yukiko Yoshimoto-Matsuda and Tomo Yoshida for excellent assistance and generous encouragement throughout the course of this study; Yumi and Yoshiomi Tamai for indispensable support; Masao Kaneki for cooperation; and Kazumi Nishihara and Dovie Wylie for expert technical assistance.

Correspondence should be addressed to Ikuo Nishimoto, Department of Pharmacology and Neurosciences, KEIO University School of Medicine, Shinjuku-ku, Tokyo 160-8582, Japan. E-mail: nisimoto@mc.med.keio.ac.jp.

Copyright (C) 2001 Society for Neuroscience $0270-6474 / 01 / 216597-11 \$ 15.00 / 0$ body treatment causes death in neuronal cells. Consistently, considerable amounts of APP are found on the surface of neurons (Jung et al., 1996; Brouillet et al., 1999; Sudo et al., 2000).

Parallel to these studies on wt APP, FAD-associated V642 mutants of APP have been shown to induce neuronal apoptosis (Wolozin et al., 1996; Yamatsuji et al., 1996a; Zhao et al., 1997; Luo et al., 1999). Toxicity by FAD mutants of APP and presenilin (PS)-2 is most likely a controllable process, mediated by pertussis toxin (PTX)-sensitive G-proteins (PG) (Wolozin et al., 1996; Yamatsuji et al., 1996a,b; Giambarella et al., 1997; Hashimoto et al., 2000). Furthermore, V642I-specific toxicity does not occur when APP lacks the His ${ }^{657}$-Lys ${ }^{676}$ region, termed Domain 20 (Yamatsuji et al., 1996a,b; Hashimoto et al., 2000). Okamoto et al. (1995, 1996) showed in vitro that wt APP and V642I-APP exert antibody-dependent and constitutive PG-activating functions, respectively, both through Domain 20. Sudo et al. (2000) confirmed that cell-surface wt APP triggers neuronal death antibody dependently in a PTX-sensitive manner. These findings suggest that APP, through PG, performs signaling functions mediated by Domain 20.

On the other hand, the study of Murayama et al. (1996) suggested that wt APP could also trigger PTX-resistant signaling pathways. Consistently, Hashimoto et al. (2000) found that FADassociated NL mutant of APP not only exerted PTX-sensitive toxicity through Domain 20 but also exerted PTX-resistant neurotoxicity through the Met ${ }^{677}-\mathrm{Asn}^{695}$ region, termed Domain 19. These findings suggest that APP has a PG-independent signaling 
function mediated by Domain 19. In accord with this idea, Lu et al. (2000) found that the Ala ${ }^{665}-\mathrm{Asn}^{695}$ peptide, the C-terminal fragment generated through APP cleavage by caspases, is cytotoxic. This study was thus conducted to investigate how the $\mathrm{C}$ terminus of APP can contribute to the regulation of intracellular signals. Here we report a novel function of APP to interact with JIP-1b/IB1, which provides the scaffold with c-Jun N-terminal kinase (JNK).

\section{MATERIALS AND METHODS}

Oligonucleotides. The nucleotide sequences of oligonucleotides used were as follows: SM60, 5' -aattttacccatatgatgtgccagattatgcctctcccgaattcggatccc3'; SM61, 5' -tcgagggatccgaattcgggagaggcataatctggcacatcatatgggtaa-3'; SM71, 5' -gatccgaattcgcggcegcgtcgactctagactcgagaagctt-3'; SM72, 5'-aattaagcttctcgagtctagagtcgacgcggccgcgaattcg-3'; SM74, 5'-ggccgaattccgcaagaagaaaccctatgg-3'; SM78, 5'-agctccgccatgggatacccttatgatgtgccagattatgccggatccccggaattc-3'; SM79, 5'-ctaggaattccggggatccggcataatctggcacatcataagggtatcccatggcgg-3'; SM108, 5' -ggccgaattcaagatggatgcagagttcggacatgattcaggatttgaag-3'; SM109, 5'-ggccgaattcaagaagaaacagtacacatccatcc3'; SM110, 5'-ggccctcgagttagttctgcatttgctcaaagaacttg-3'; SM121, 5'-ggccgaattcaggaagaggcagtacgg- $3^{\prime} ;$ SM122, 5' -ggccctcgagttaaatctgcatctgctccagg-3'; SM123, 5' -ggccctcgagtcagttctgctgcatcttggag-3'; SM124, 5' -gaattcaagaagaaacaggccacatccatccatcatg-3'; SM125, 5'-cgccgccgtggaaccagaggagc-3'; SM126, 5'-gatgcagcagaacggaggtgagaatccaacttac-3'; SM127, 5'gcagaacggatatgaggctccaacttacaagttc-3'; SM132, 5'-cggatatgagaatccaactgccaagttctttgagc-3'; SM138, 5'-ggccgaattcagcgactggattgaccag-3'; SM139, $5^{\prime}$-ggccctcgagctactccaagtagatatcttctg-3'; SM143, 5'-gcagcccttgccaaaaacagctgtgtccttgagatcag-3'; SM241, 5' -gcctcgagtcaagcatccagacacttctggt-3'; and SM242, 5' -gcggatccttcgctgtgcgctcc- $3^{\prime}$.

Plasmid construction. The sequences of all constructs were verified by dideoxy-termination using ABI310 sequencer (PE Applied Biosystems, Foster City, CA). PCR inserts were amplified by deep vent DNA polymerase (New England Biolabs, Beverly, MA) or KOD DNA polymerase (Toyobo, Osaka, Japan). Mouse brain cDNA used for RT-PCR was prepared from total RNA of ICR adult mouse, isolated using an RNeasy kit (Qiagen GmbH, Hilden, Germany) and reverse transcribed using Superscript II and an Oligo-dT primer (Life Technologies Oriental, Tokyo, Japan). pEG202-NLS polylinker portion was exchanged with the corresponding EcoRI-XbaI fragment of pEG202; then hemagglutinin (HA) epitope, encoding YPYDVPDYA, was inserted between EcoRI and XhoI using linkers SM60/SM61 (pEG202-NLS-HA). The coding sequences of APP, APLP1, and APLP2 and their variants were PCR amplified and cloned into pEG202 and pEG202-NLS-HA using EcoRI/ $X$ XhoI. APP fragments were amplified from mouse $\mathrm{APP}_{695}$ with the following primers: $\mathrm{APP}_{649-695}$, SM109/SM110; $\mathrm{APP}_{595-695}$, SM108/ SM110; and APP ${ }_{649-680}$, SM109/SM123. The cytoplasmic domain of APLP1 (APLP1 ${ }_{607-653}$ ) was amplified with PCR from APLP1 plasmid obtained in another two-hybrid screening using SM74. The cytoplasmic domain of APLP2 ${ }_{751}$ (APLP2 ${ }_{705-751}$ ) was cloned by RT-PCR using primers SM121/SM122. The point mutants of cytoplasmic domain fragments of APP were constructed by PCR-directed mutagenesis of $\mathrm{APP}_{649-695}$ and $\mathrm{APP}_{649-680}$ and cloned into pEG202 and pEG202NLS-HA using the following primers: Y653A, SM124; T668E, SM125; Y682G, SM126; N684A, SM127; and Y687A, SM132, respectively. Among the prey fusion plasmids used in the interaction mating, JIP-1 493-660 was PCR constructed from JIP-1b H93-707 $_{\text {using SM143. }}$ JIP-1 $\mathrm{b}_{557-707}$ was PCR constructed using primes SM138/SM139. Other prey fusions were directly cloned in the screening. All prey fusions were cloned into EcoRI/XhoI of pJG4-5.

JIP-1b was cloned by RT-PCR and cloned into KpnI/XbaI of pcDNA3.1-HisA (Invitrogen, Carlsbad, CA) (T7-JIP-1b). JIP-1 constructs were created using the prey fusion of JIP-1 $1_{493-660}$ and T7-JIP-1b. T7-JIP- $1 \mathrm{~b}_{1-272}$ was constructed by deleting XhoI fragment from T7-JIP1b. In constructing T7-JIP $1_{271-660}$, T7-JIP-1 365-707, and T7-JIP-1 $_{365-660}$, corresponding fragments were cloned into pcDNA3.1-HisC (Invitrogen). pcDNA3 (Invitrogen) was modified using linkers SM78/SM79 and SM71/ SM72 to insert Kozak's consensus sequence followed by HA epitope MGYPYDVPDYAGSP. IB1 ${ }_{360-711}$ was cloned into the EcoRI/XhoI site of this vector (HA-IB1 $1_{360-711}$ ). pGEX-2T (Amersham Pharmacia Biotech, Uppsala, Sweden) polylinker was modified by the insertion of the linker SM71/SM72, using BamHI/EcoRI (pGEX-2Tm). Glutathione $S$-transferase (GST)-fusion proteins used for bacterial protein expression were constructed by cloning the same PCR inserts used for yeast bait vectors in $E c o$ RI/XhoI of pGEX-2Tm. Full-length mouse wt APP and its FAD mutants were cloned into pEF-BOS (Mizushima and Nagata, 1990).

Corresponding portions of IB1, JIP-1b, and JIP-1 were cloned into pEBG (Sanchez et al., 1994), a mammalian expression vector of GST, in constructing GST-IB1 $1_{360-711}$, GST-JIP-1b $\mathrm{b}_{493-707}$, and GST-JIP-1b $\mathrm{b}_{540-707}$ pEBG without insert was used as GST control. GST-JNK1 $\beta 1$ was constructed by cloning the $B g l$ II fragment of SRHis-JNK1 $\beta 1$ (Hirai et al., 1996) into the BamHI site of pEBG.

Corresponding portions of JIP-1b, mDab1, and X11, obtained in the two-hybrid screening, were cloned into pET28(a) (JIP-1b and mDab1) or pET28(c) (X11) (Novagen, Madison, WI). The region containing two PIDs of Fe65 was PCR amplified from a Fe65 clone obtained in the screening using SM241/SM242 and cloned into pET28(a). These pET constructs were used to produce His-tagged JIP- $1 \mathrm{~b}_{493-707}$, His-tagged $\mathrm{mDab}_{2-217}$, Histagged $\mathrm{X} 11_{37-680}$, and His-tagged $\mathrm{Fe}_{370-662}$, respectively.

Antibodies. Anti-T7 antibody (Novagen), anti-HA antibody (12CA5: Roche Diagnostics, Basel, Switzerland), anti-APP antibody (22C11: Roche Diagnostics), and anti-GST antibody (MAB2510: Upstate Biotechnology, Lake Placid, NY) were used for immunoblotting. Rabbit antisera were raised for CGGAASPPAASPFLGLHIASPPNFR corresponding to residues $10-33$ of JIP-1b ( $\alpha \mathrm{JIPN})$ and RAFQQFYKQFVEYTCPTEDIYLE corresponding to residues 685-707 of JIP-1b ( $\alpha$ JIPC).

Yeast two-hybrid method. A LexA yeast two-hybrid system was purchased from OriGene Technologies (Rockville, MD). Yeast two-hybrid screening was performed according to standard procedures (Ausubel et al., 1999), using the yeast transformation method of Gietz and Schiestl (1995). All bait constructs of APP and its mutants failed to activate LEU2 or LacZ reporters. EGY48 (MAT $\alpha$, trp1, his3, ura3, leu2::6 opsLEU2) was used for the screening with pEG202-NLS-HA$\mathrm{APP}_{595-695}$, and EGY188 (MAT $\alpha$, trp1, his3, ura3, leu2::2 ops-LEU2) was used for pEG202-NLS-HA-APP ${ }_{649-695}$. pSH18-34 (Ausubel et al., 1999) was used for the LacZ reporter. The positive control bait was pSH17-4 (GAL4 activation domain fused to LexA), and the negative control baits were pRFHM1 (homeodomain of bicoid fused to LexA) and pEG202Max (Max fused to LexA), provided in the kit. The human adult AD patient library was constructed using the method of Gubler and Hoffman (1983), using cDNA obtained from a brain of a patient diagnosed as having AD by necropsy, according to institutional guidelines. The mouse adult brain library was purchased from Clontech (Palo Alto, CA). Yeast strains were cultured on YPAD plates, or on minimal plates with these combinations of the following conditions: containing glucose (SD plates), or containing galactose and raffinose (Gal plates), or containing 5-bromo-4-chloro-3-indolyl- $\beta$-D-galactopyranoside (X-gal), or lacking the combinations of histidine (-his), uracil (-ura), tryptophan (-trp), or leucine (-leu), as described in Ausubel et al. (1999). To extract yeast proteins, cells with the same optical density were collected and suspended with $2 \times$ sample buffer, frozen, and then boiled, according to the protocol of Ausubel et al. (1999). Yeast interaction mating was performed according to the methods of Finley and Brent (1994) and Meroni et al. (1997). Briefly, the bait constructs were transformed into RFY206 (MATa, trp1 $::$ hisG, his3 200 , ura3-52, leu2-3, lys2 201 ) simultaneously with the LacZ reporter and grown as patches on SD-his-ura plates. The various fragments of genes cloned into pJG4-5 prey vectors were transformed into EGY48 and grown as lawns on SD-trp plates. The mating was performed as follows. On the first day, the patches of RFY206 bait transformants and the lawns of EGY48 prey transformants were replica plated on the same YPAD plates. The next day, the mated yeast on the YPAD plates was replica plated on SD-his-ura-trp-leu and Gal-his-ura -trp-leu plates and incubated for an additional $48 \mathrm{hr}$ to monitor cell growth reflecting galactose-induced LEU2 reporter activity, and on SD-his-ura-trp plates to select the mated yeast. On the third day, the patches grown on the replica plated SD-his-ura-trp plates were again replica plated on SD (X-gal)-his-ura-trp and Gal (X-gal)-his-ura-trp plates and incubated an additional $24 \mathrm{hr}$ to monitor galactose-induced LacZ reporter activity.

Bacterial protein production and purification. GST fusion proteins were expressed using BL21-Gold (Stratagene, La Jolla, CA) and purified with glutathione Sepharose 4B (Amersham Pharmacia Biotech), according to the manufacturer's protocol. The purified proteins were dialyzed three times against $100 \mathrm{vol}$ of buffer A (20 mM Tris/HCl, pH 8.0, 1 mm DTT, $1 \mathrm{~mm}$ EDTA), and frozen at $-80^{\circ} \mathrm{C}$ until use. His-tagged proteins were expressed using BL21(DE3) (Novagen) and purified with Chelating Sepharose Fast Flow (Amersham Pharmacia Biotech) loaded with 
$\mathrm{NiSO}_{4}$, according to the manufacturer's protocol. The purified proteins were dialyzed against buffer $\mathrm{A}$ and frozen until use, as above.

Transfection and cell lysate. COS7 cells $\left(1 \times 10^{6}\right)$ were seeded in $10 \mathrm{~cm}$ dishes the day before the transfection in high-glucose DMEM supplemented with $10 \%$ fetal bovine serum (FBS), $100 \mathrm{U} / \mathrm{ml}$ of penicillin, and $100 \mu \mathrm{g} / \mathrm{ml}$ of streptomycin sulfate. The cells were transfected with $6 \mu \mathrm{g}$ of DNA using DEAE-dextran. NT2 cells (Stratagene), human neuronal precursor cells, were seeded at $1 \times 10^{6}$ in $10 \mathrm{~cm}$ dishes the day before the transfection in 50\% DMEM, 50\% Ham's F-12 supplemented with $10 \%$ FBS, $100 \mathrm{U} / \mathrm{ml}$ of penicillin, $100 \mu \mathrm{g} / \mathrm{ml}$ of streptomycin, and $2 \mathrm{~mm}$ L-glutamine. They were then transfected with $12 \mu \mathrm{g}$ of DNA using LipofectAMINE (Life Technologies Oriental).

Forty-eight hours after the initiation of transfection, the cells were washed once with PBS, lysed for $30 \mathrm{~min}$ at $4^{\circ} \mathrm{C}$ in buffer B [20 mM HEPES/NaOH, pH 7.4, 1 mM DTT, 1 mM EDTA, $150 \mathrm{~mm} \mathrm{NaCl}, 0.5 \%$ (w/v) Triton X-100], supplemented with $10 \%$ (v/v) glycerol, $1 \mathrm{~mm}$ phenylmethylsulfonylfluoride, $10 \mu \mathrm{g} / \mathrm{ml}$ of aprotinin, and $10 \mu \mathrm{g} / \mathrm{ml}$ of leupeptin with occasional gentle shaking. The lysed cells were collected and centrifuged at $20000 \times g$ for $15 \mathrm{~min}$, and cleared lysates were used for further analysis.

Bacterial GST pull-down. In pull-down experiments using His-tagged proteins, $6 \mu \mathrm{g}$ of GST-fusion protein was incubated with $20 \mu \mathrm{l}$ of glutathione beads in a total volume of $0.5 \mathrm{ml}$ of buffer $\mathrm{A}$ for $1 \mathrm{hr}$ at $4^{\circ} \mathrm{C}$ and washed once with buffer $\mathrm{C}$ [20 mM Tris/HCl, pH 7.4, 1 mm DTT, 1 mM EDTA, $150 \mathrm{~mm} \mathrm{NaCl}, 0.1 \%$ (w/v) Triton X-100]. His-tagged proteins of various concentrations were adjusted to $150 \mathrm{~mm} \mathrm{NaCl}$ and $0.1 \%$ Triton X-100, mixed with beads immobilizing GST-fusion protein for 2 hr at $4^{\circ} \mathrm{C}$ with rotation, and washed three times with buffer $\mathrm{C}$. The washed beads were mixed with $90 \mu \mathrm{l}$ of $1 \times$ sampling buffer, boiled, and subjected to immunoblotting and Coomassie brilliant blue (CBB) staining. In the pull-down experiments using COS cell lysate, $30 \mu \mathrm{g}$ of GST fusion proteins were immobilized on $30 \mu \mathrm{l}$ of glutathione beads as above, and washed once with buffer B. Lysates ( $0.5 \mathrm{mg}$ protein) were incubated with the beads immobilizing GST-fusion protein for $2 \mathrm{hr}$ at $4^{\circ} \mathrm{C}$ with rotation, and washed three times with buffer $\mathrm{B}$. The washed beads were mixed with $90 \mu \mathrm{l}$ of $1 \times$ sample buffer, boiled, and subjected to further analysis as above.

Immunoprecipitation. Cell lysates ( $0.5 \mathrm{mg}$ protein) were incubated with $\alpha \mathrm{JIPN}$ or $\alpha \mathrm{JIPC}$ or corresponding preimmune sera for $1 \mathrm{hr}$ at $4^{\circ} \mathrm{C}$, and mixed with $10 \mu \mathrm{l}$ of Protein G-Sepharose 4 Fast Flow (Amersham Pharmacia Biotech) for an additional $1 \mathrm{hr}$ with rotation at $4^{\circ} \mathrm{C}$. Immunocomplexes were washed three times with buffer $\mathrm{B}$, boiled in $1 \times$ sample buffer, and subjected to immunoblotting.

Immunoblotting. Protein samples were submitted to SDS-PAGE, transferred to nitrocellulose membranes (BA85; Schleicher \& Schuell, Dassel, Germany) using a semidry system, and blocked overnight with TBS (50 $\mathrm{mm}$ Tris/ $\mathrm{HCl}, \mathrm{pH} 7.5,200 \mathrm{~mm} \mathrm{NaCl})$ plus 5\% skim milk. The membranes were incubated $1 \mathrm{hr}$ at room temperature with the primary antibodies or antisera diluted in the same buffer, and then washed five times with TBS containing $0.05 \%$ Tween 20 . The secondary antibodies, horseradish peroxidase-conjugated anti-rabbit goat polyclonal antibody (Bio-Rad, Hercules, CA) and horseradish peroxidase-conjugated antimouse rabbit polyclonal antibody (Bio-Rad), were diluted in TBS containing 5\% skim milk. After washing, the membranes were visualized using an ECL kit (Amersham Pharmacia Biotech) and developed using x-ray films (Fujifilm, Tokyo, Japan).

Cell staining. COS cells $\left(3 \times 10^{5}\right)$ were seeded in $60 \mathrm{~mm}$ dishes on the previous day and transfected with $2 \mu \mathrm{g}$ of plasmids using LipofectAMINE (Life Technologies Oriental), and reseeded on coverslips the next day. Forty-eight hours after the initiation of the transfection, cells were fixed with $0.1 \mathrm{M}$ phosphate buffer, $\mathrm{pH} 7.4$, containing $4 \%$ paraformaldehyde for $20 \mathrm{~min}$ at room temperature. After extensive washing with PBS, the fixed cells were incubated with $\alpha$ JIPN, $\alpha$ JIPC, preimmune sera, anti-APP antibody (22C11), or mouse IgG (Cortex Biochem, San Leandro, CA) in PBS containing 3\% BSA and $0.1 \%$ Triton $\mathrm{X}-100$ overnight at $4{ }^{\circ} \mathrm{C}$, and visualized by goat fluorescein isothiocyanate (FITC)-conjugated anti-rabbit IgG or horse Texas Red-conjugated antimouse $\operatorname{IgG}$ (Vector Laboratories, Burlingame, CA) in PBS containing $3 \%$ BSA. DNA was stained by incubating coverslips in PBS containing $0.2 \mu \mathrm{M} \quad 2^{\prime}$-[4-ethoxyphenyl]-5-[4-methyl-1-piperazinyl]-2,5'-bi-1Hbenzimidazole (Hoechst 33342; Sigma-Aldrich Japan, Tokyo, Japan), and washed five times with PBS. Coverslips were mounted to slides with Vectashield (Vector Laboratories). Digital images were taken with an LSM310 laser scanning microscope (Carl Zeiss, Jena, Germany).
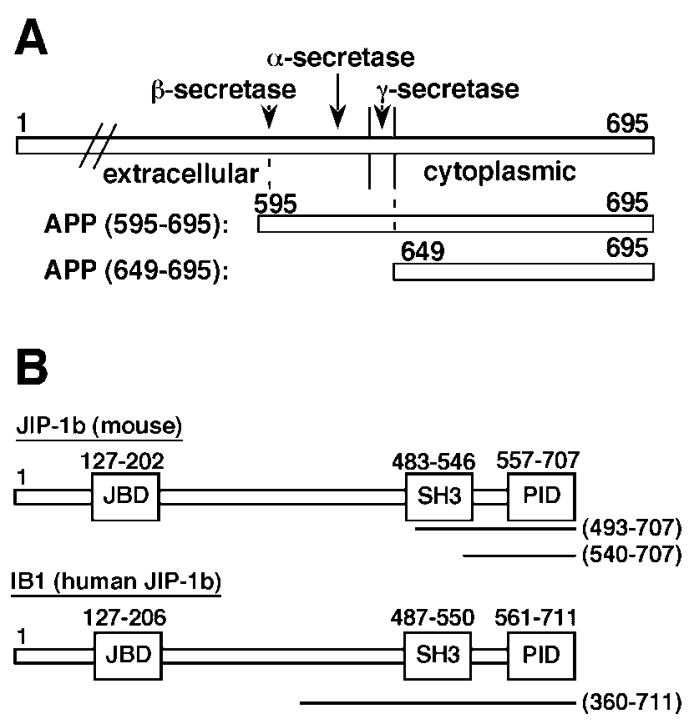

Figure 1. The bait used and clones recovered in the two-hybrid screening. $A$, An illustration of the regions of mouse $\mathrm{APP}_{695}$ used as bait. $\mathrm{APP}_{595-695}$ starts from the two amino acids Lys ${ }^{595}{ }_{-\mathrm{Met}^{596}}$, mutated to Asn-Leu in the Swedish type of FAD. APP ${ }_{649-695}$ encodes the entire cytoplasmic domain of APP, the amino acid sequence of which is conserved between human and mouse APP. The $\alpha$-, $\beta$-, and $\gamma$-secretase cleavage sites are also indicated. $B$, The clones recovered from the screening and the domain organization of mouse JIP-1b and its human homolog IB1. The portions coded by the recovered plasmids are indicated as lines with their amino acid positions in parentheses. The boxes indicate JNK binding domain $(J B D)$, src-homology region 3 (SH3), and PID, with their amino acid positions in numbers.

\section{RESULTS}

\section{Screening of molecules interactive with cytoplasmic domains of APP}

Twenty million transformants of an adult mouse brain library were screened with the transmembrane plus cytoplasmic domain $\mathrm{APP}_{595-695}$ and the cytoplasmic domain $\mathrm{APP}_{649-695}$ (Fig. 1A). All retransformation-positive clones carried a PID (Kavanaugh and Williams, 1994; Bork and Margolis, 1995; Kavanaugh et al., 1995) that was implicated in the binding to the NPXY motif. In addition to known APP-binding proteins-such as Fe65 (Fiore et al., 1995), Fe65L (Guenette et al., 1996), Fe65L2 (Duilio et al., 1998), X11 (Borg et al., 1996), and mDab1 (Trommsdorff et al., 1998) - a novel APP-interacting protein was found to be JIP-1b (Whitmarsh et al., 1998; Kim et al., 1999), a PID-containing form of JIP-1 (Dickens et al., 1997).

Because the recovered plasmids from the two different series of screening were almost identical, and $\mathrm{APP}_{649-695}$ showed stronger interaction with PID-containing clones than $\mathrm{APP}_{595-695}$ in a two-hybrid assay (data not shown), the human adult brain library of an AD patient was screened only with $\mathrm{APP}_{649-695}$. After screening 14 million transformants, we found three interacting molecules: Fe65, X11L (Tomita et al., 1999), and IB1 (Bonny et al., 1998). X11L is the homolog of mouse X11 (Tomita et al., 1999), and IB1 is the human homolog of mouse JIP-1b (Bonny et al., 1998). Because JIP-1b and IB1 were novel APP-interacting proteins, and cloned from both the adult mouse brain library and the human patient brain library, we further analyzed JIP-1b/IB1 in this study. The regions encoded by the recovered JIP-1b/IB1 plasmids are indicated in Figure $1 B$. 


\section{Requirement of PID in JIP-1b/IB1 and $Y^{682}, N^{684}$, and $Y^{687}$ in $G^{681}$ YENPTY ${ }^{687}$ of APP}

To determine the critical amino acids necessary for interaction of JIP-1b/IB1 with the APP cytoplasmic domain, various APP mutants (Fig. 2A) were constructed in both pEG202 and pEG202NLS-HA bait vectors and transformed into RFY206 with LacZ reporter pSH18-34. When the transformants of pEG202-NLS-HA fusions were submitted to immunoblot analysis using anti-HA antibody, a single immunoreactive band corresponding to the expected size of the bait fusions was detected in each lane of transformants (Fig. 2B). Various JIP-1b/IB1 fusions in the prey vector pJG4-5 (Fig. 2D) were transformed into an EGY48 yeast strain. The array of two patches of each RFY206 bait transformant harboring the bait constructs (Fig. 2C) was mated with the lawns of EGY48 prey transformants, and the mated cells were grown on minimal medium containing glucose or galactose and lacking histidine, uracil, tryptophan, and leucine, to assay galactose-induced LEU2 reporter activity, as shown in Figure $2 D$.

The fusions of $\mathrm{IB}_{360-711}, \mathrm{JIP}-1 \mathrm{~b}_{493-707}, \mathrm{JIP}-1 \mathrm{~b}_{540-707}$, and JIP-1 $b_{557-707}$, all of which contain intact PID, interacted with $\mathrm{APP}_{649-695}$ (cyt) and its Y653A and T668E mutants [cyt (Y653A), cyt (T668E)]. In contrast, no interaction was observed with the other mutants of $\mathrm{APP}_{649-695}$ [cyt (Y682G), cyt (N684A), and cyt (Y687A)] or $\mathrm{APP}_{649-680}(\Delta \mathrm{NPTY})$, nor with any of its mutants [ $\Delta \mathrm{NPTY}$ (Y653A), $\Delta \mathrm{NPTY}(\mathrm{T} 668 \mathrm{E})]$ (Fig. 2D). This indicates that (1) the extreme $\mathrm{C}$-terminal 15 amino acids of APP harboring $\mathrm{G}^{681} \mathrm{YENPTY}^{687}$ are necessary for the interaction of JIP-1b/IB1 with APP; (2) Tyr ${ }^{682}$, Asn ${ }^{684}$, and $\mathrm{Tyr}^{687}$, contained in this region, are all critical for this interaction; and (3) the 151 amino acid PID region contained in the shortest construct, JIP$1 b_{557-707}$, is sufficient for the interaction with APP. In further support of this observation, the fusion of JIP- $1_{493-660}$, which is equivalent to the JIP-1b $\mathrm{b}_{493-707}$ lacking 47 amino acids in the PID region, exhibited no interaction with the cytoplasmic domain of wt APP or APP mutants (Fig. 2D). This observation suggests that the PID region was sufficient and necessary for the interaction of JIP-1b with APP.

Other PID-containing fusions obtained in the same screening were also examined for their ability to interact with cytoplasmic domain mutants of APP, as shown in Figure 2D. Fe65 $254-708$, $\mathrm{X} 11_{37-680}$, and $\mathrm{mDab}_{2-217}$ all displayed $\mathrm{G}^{681} \mathrm{YENPTY}^{687}$ dependent interaction with the cytoplasmic domain of APP, as evidenced by their positive interaction with cyt, cyt (Y653A), and cyt (T668E), and their negative interaction with $\triangle \mathrm{NPTY}$, $\Delta$ NPTY (Y653A), and $\Delta$ NPTY (T668E) (Fig. 2D). However, the critical APP residues necessary for the interactions were different in the three constructs. Fe $65_{254-708}$ required $\mathrm{Tyr}^{682}$, but not Asn ${ }^{684}$ or $\mathrm{Tyr}^{687}$; X11 $137-680$ required $\mathrm{Tyr}^{682}$ and Asn ${ }^{684}$, but not $\mathrm{Tyr}^{687}$; $\mathrm{mDab}_{2-217}$ required $\mathrm{Tyr}^{682}$ and $\mathrm{Asn}^{684}$, as well as $\mathrm{Tyr}^{687}$, the same residues necessary for JIP-1b/IB1 constructs containing PID (Fig. 2D). Therefore, in this experiment, the mode of APP interaction with JIP-1b/IB1 was distinct from that with Fe65 and X11, but indistinguishable from that with mDab1.

These observed interactions were confirmed by replica-plating the mated yeast cells to minimal plates, containing X-gal, and containing glucose or galactose, and lacking histidine, uracil, and tryptophan. The LacZ reporter activity visualized by the blue color development displayed the same patterns as in Figure $2 D$ (data not shown). The same set of cytoplasmic domain mutants of APP fused to pEG202 bait vector displayed the same results as those of pEG202-NLS-HA fusion constructs (data not shown).
These data indicate that JIP-1b/IB1 interacts with APP in a manner distinct from already known interactions of Fe65 or X11 with APP, and both PID of JIP-1b/IB1 and the $\mathrm{G}^{681}{ }^{\mathrm{Y} E N P T Y}{ }^{687}$ region of APP, especially $\mathrm{Tyr}^{682}, \mathrm{Asn}^{684}$, and $\mathrm{Tyr}^{687}$, are essential for the interaction.

\section{Purified JIP-1b protein binds to the cytoplasmic domain of APP with affinity comparable to that of mDab1, X11, and Fe65}

To compare the binding affinity of JIP-1b PID with that of other PID-containing proteins, His-tagged JIP-1 $\mathrm{b}_{493-707}$, which contains a part of SH3 domain and the entire PID (the same region illustrated in Fig. 2D), was expressed in bacteria, purified, and subjected to pull-down experiments using bacterially produced recombinant APP cytoplasmic domain fused to GST (GST-cyt). His-tagged JIP-1b at various concentrations was mixed with glutathione beads immobilizing an equivalent amount of GSTcyt, and each precipitant (ppt) was probed for GST-cyt by CBB staining (Fig. 3A, top panel), and for the bound His-tagged protein by immunoblotting using anti-T7 antibody (Fig. $3 A$, bottom panel). A similar amount of GST-cyt was detected in all precipitants (Fig. $3 A$ ). When the bound His-tagged JIP-1b was plotted against the concentration used for the binding experiment, the bound protein showed saturable binding to GST-cyt with half-maximal binding at $\sim 0.4 \mu \mathrm{M}$ (Fig. $3 A$ ). His-tagged JIP-1b did not bind to GST at all concentrations tested, indicating that the binding of His-tagged JIP-1b to GST-cyt is specific (data not shown).

Similar experiments were performed using His-tagged $\mathrm{mDab}_{2-217}$ (the same region illustrated in Fig. $2 D$ ), His-tagged X11 ${ }_{37-680}$ (the same region illustrated in Fig. 2D), and His-tagged Fe65 $370-662$ (the region containing its two PIDs). Again, a similar amount of GST-cyt was detected in all precipitants (Fig. 3B-D, top panels). When the bound PID-containing proteins were plotted against their concentration used for the binding, the binding curves showed saturable binding with the concentration of mDab1, X11, and Fe65 required for their half-maximal binding at $\sim 0.5,0.5$, and $0.15 \mu \mathrm{M}$, respectively (Fig. $3 B-D$ ). None of these His-tagged proteins bound to GST at any of the concentrations tested, which confirmed the specificity of their binding (data not shown). The purity of the recombinant proteins used for these binding experiments as revealed by SDS-PAGE and CBB staining is shown in Figure $3 E$.

Therefore, bacterially expressed and purified JIP-1b bound to the cytoplasmic domain of APP in a specific and saturable manner, and it showed half-maximal binding at a submicromolar concentration, which is comparable with the half-maximal binding concentrations of other PID proteins-mDab1, X11, and Fe65. In addition, the data that purified recombinant JIP-1b bound to purified GST-cyt suggest that JIP-1b directly binds to the cytoplasmic domain of APP, as mDab1, X11, and Fe65. As assessed by the common T7-antigenicity, the maximal binding of JIP-1b to the cytoplasmic domain of APP was relatively lower than that of the other PID proteins, suggesting that the APP/ JIP-1b complex might be a minority among all APP/PID-protein complexes under the conditions in which other PID proteins are also present. However, considering its comparably high affinity to those of other PID proteins, this feature of APP/JIP-1b interaction could be useful for signal transduction of high time resolution. 
A
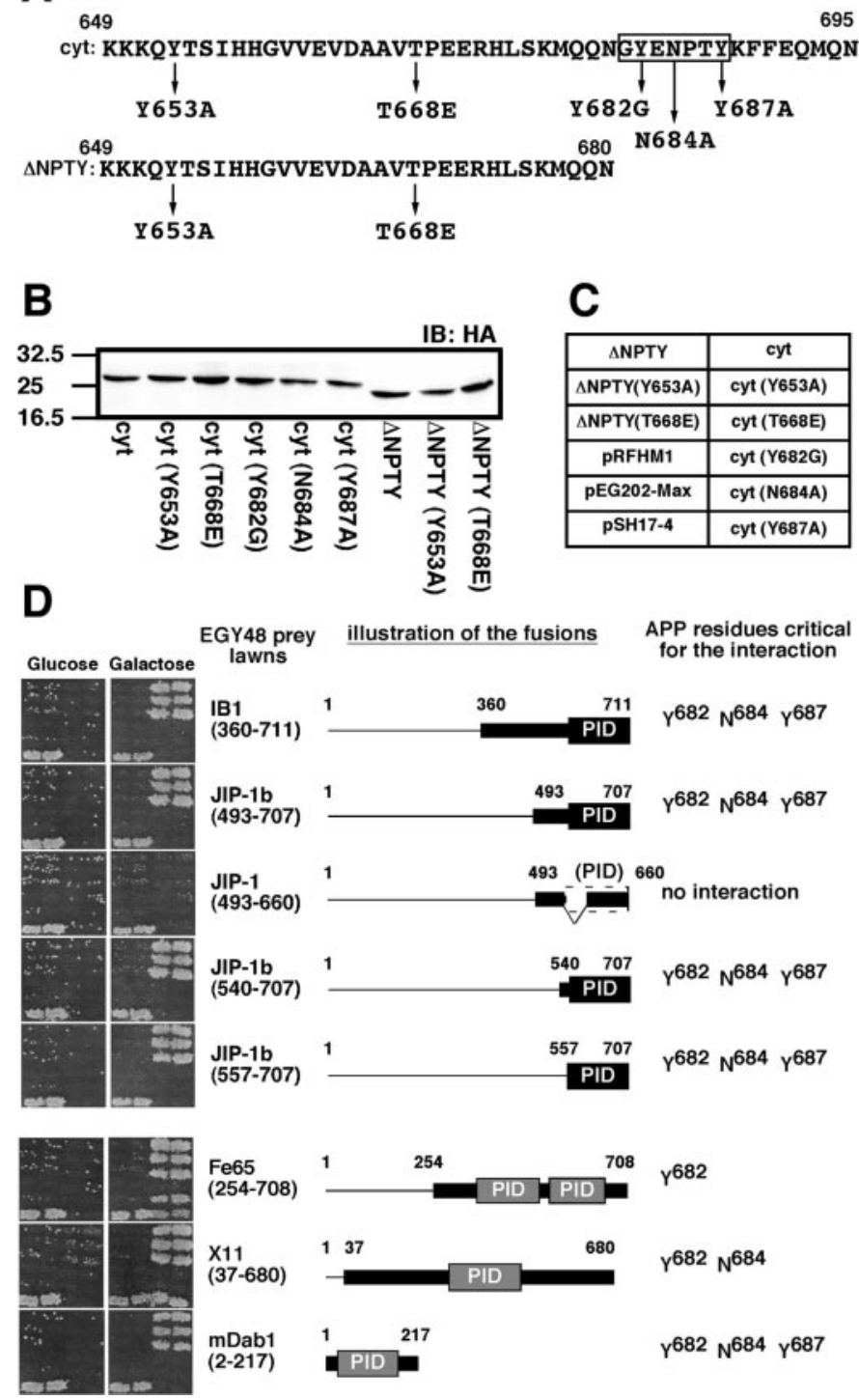

Figure 2. Requirement of both PID of JIP-1b/IB1 and $\mathrm{G}^{681}$ YENPTY 687 of APP for JIP-1b/IB1 interaction with APP. $A$, The indicated amino acid residues were changed by site-directed mutagenesis. $\mathrm{APP}_{649-695}$ and $\mathrm{APP}_{649-680}$ are designated as cyt and $\triangle \mathrm{NPTY}$, respectively. The $\mathrm{G}^{681}{ }^{6}$ ENPTY ${ }^{687}$ region is boxed. $B$, Proteins of RFY206 yeast cells transformed with fusions of various mutants of the APP cytoplasmic domain were probed with anti-HA antibody. The bait fusions used are identified under each lane. Their designations are as described in $A$. The numbers on the left are the relative molecular weights of the size markers in kilodaltons. $C$, Two patches of each bait fusion transformant of the RFY206 strain were arranged as indicated. The names of APP bait variants are described in $A$. Two negative controls ( $p R F H M 1$ and $p E G 202-M a x)$ and a positive control ( $p S H 17-4)$ are also included. $D$, Interaction-mating of APP variants and JIP-1b/IB1 or other prey fusions. The left column of pictures shows the growth of mated yeast cells on glucose plates (Glucose), and the right column shows the growth of mated yeast cells on galactose and raffinose (Galactose) plates, as described in Materials and Methods. Galactose-dependent LEU2 activity was displayed by the difference in the growth of the mated cells between that in the glucose plates, which suppress the expression of the prey fusions, and that in the galactose plates, which induce it. The two negative controls (pRFHM1, pEG202-Max) showed no growth in any combination. The prey fusions are indicated at the right of the columns. The illustration of the fusions indicates the regions coded by the cDNA and the constructs as thin and thick lines, respectively. The first and last amino acids of cDNA and regions encoded by the constructs are indicated, except for $m D a b 1_{2-217}$, which starts at the second codon. PID regions were indicated as black
A
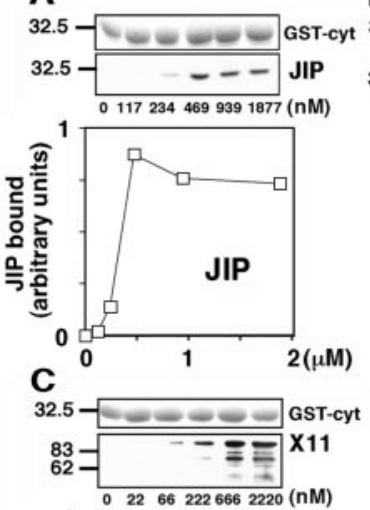

B

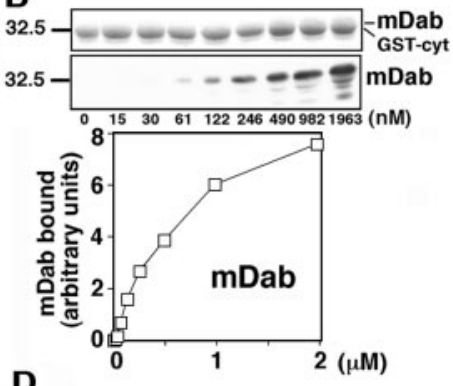

D
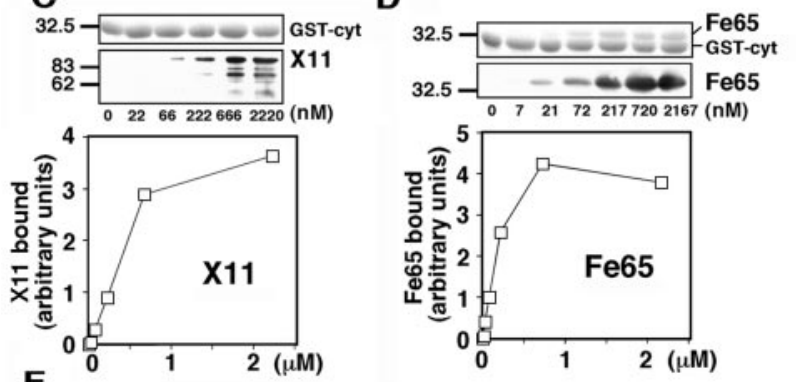

Figure 3. Purified JIP-1b binds to the cytoplasmic domain of APP with affinity comparable to those of other PID-containing proteins. A, Histagged JIP-1 $\mathrm{b}_{493-707}$, at the concentrations indicated under each lane, was precipitated with glutathione beads immobilizing the APP cytoplasmic domain fused to GST (GST-cyt). Precipitated GST-cyt was visualized with CBB staining (top panel), and bound His-tagged JIP- $1 \mathrm{~b}_{493-707}$ in the precipitants was detected with anti-T7 antibody (bottom panel). The numbers on the left are the relative molecular weights of the size markers in kilodaltons. The bound JIP-1b, calculated from the pixels of scanned image and a standard curve, was plotted against the concentration of JIP-1b used for the binding. $B$, Similar experiments were performed using His-tagged $\mathrm{mDab}_{2-217}$. The bands corresponding to $\mathrm{mDab} 1$ are faintly visible in $\mathrm{CBB}$ staining (top panel). $C$, Similar experiments were performed using Histagged X11 $137-680 . D$, Similar experiments were performed using His-tagged Fe65 $370-662$. The bands corresponding to Fe65 are visible in CBB staining (top panel). E, CBB staining of the purified His-tagged proteins used.

\section{C terminus of APP precipitates JIP-1b/IB1 in a $G^{681}$ YENPTY $^{687}$-dependent manner}

To confirm the protein interaction of JIP-1b/IB1 with APP, the lysate of COS cells transfected with HA-IB1 $1_{360-711}$ were mixed with various GST fusion proteins immobilized on glutathione beads, and the lysate and precipitants were analyzed by immunoblotting using anti-HA antibody (Fig. $4 B$ ). HA-IB1 $1_{360-711}$ was detected in the precipitant of GST fused with the cytoplasmic domain of APP (GST-cyt), but not in those of GST alone, GST fused with the cytoplasmic domain of APP lacking C-terminal 15

$\leftarrow$

boxes, except for JIP-1 ${ }_{493-660}$, which has incomplete PID. APP residues critical for the interaction are noted at the right side, except for JIP- $1_{493-660}$, which displayed no interaction with any APP variants. The GenBank accession numbers and amino acids corresponding to the PIDs were IB1, NM005456, 557-711; JIP-1b, AF054611, 557-707; JIP-1, AF003115, no PID; Fe65, AF206270, 364-505 and 535-660; X11, L34676, 297-460; mDab1, Y08381, 37-175. L34676 is a partial clone that lacks N-terminal 69 amino acids in its human or rat counterpart. 


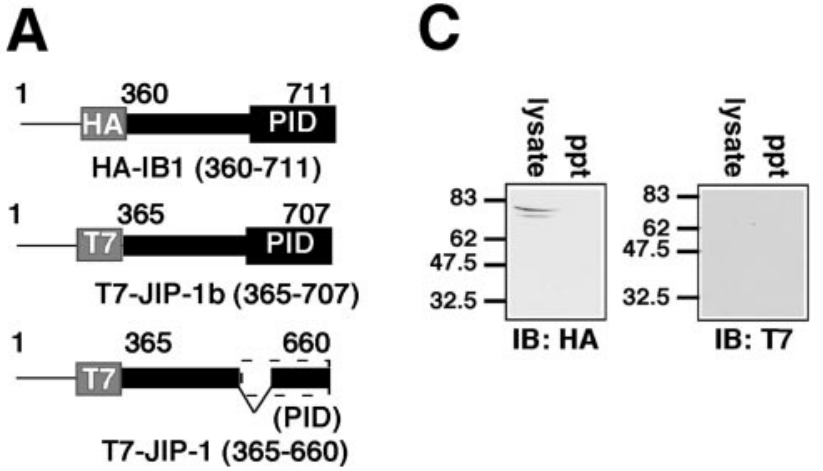

B
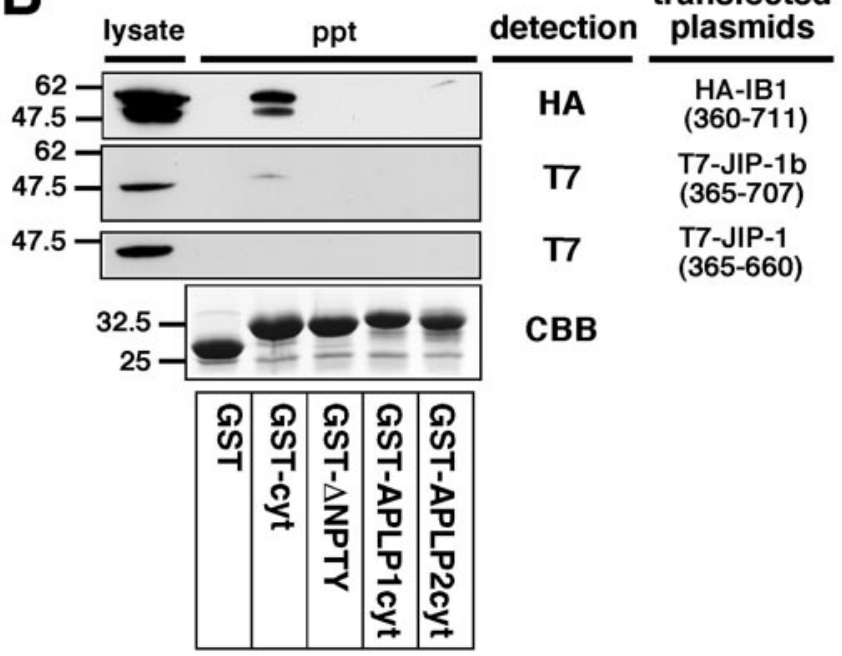

Figure 4. JIP-1b/IB1, not JIP-1, is precipitated by the cytoplasmic domain of APP, but little by those of APLP1 and APLP2. A, An illustration of the JIP/IB1 constructs used in the bacterial GST-fused protein pull-down experiments. HA and T7 tags are boxed. Other designations are the same as the illustration of the fusions in Figure 2D. $B$, The lysates prepared from the COS cells transfected with HA-IB1 $360-711$, T7-JIP-1 b $_{365-707}$, and T7-JIP-1 $1_{365-660}$ were precipitated with glutathione beads immobilizing GST, GST-APP ${ }_{649-695}$ (GST-cyt), GST-APP $649-680$ (GST- $\triangle N P T Y)$, GST-APLP1 $1_{607-653}$ (GST-APLP1cyt), and GSTAPLP $2_{705-751}$ (GST-APLP2cyt), and the lysate and the precipitants were visualized with anti-HA and anti-T7 antibodies as indicated. The precipitants were also stained with $\mathrm{CBB}$, which shows the presence of a similar amount of GST-fusion protein. The numbers on the left are the relative molecular weights of the size markers in kilodaltons. Each datum presented in Figure 4 is the representative of three independent experiments. $C$, The lysates prepared from mock-transfected COS cells were precipitated using GST-cyt. The lysates (lysate) and precipitants ( $p p t$ ) were visualized with anti-HA and anti-T7 antibody, indicating the absence of nonspecific bands in GST-cyt precipitants.

amino acids (GST- $\triangle$ NPTY), or GST fused with the cytoplasmic domain of APLP1 (GST-APLP1cyt). Minor immunoreactive bands of lower relative molecular weights were attributed to partial degradation of the expressed protein. A faint band was detected occasionally in the precipitant of GST fused with the cytoplasmic domain of APLP2 (GST-APLP2cyt) (Fig. 4B). It is thus highly likely that $\mathrm{IB}_{360-711}$ binds to the cytoplasmic domain of APP, which contains $\mathrm{G}^{681} \mathrm{YENPTY}^{687}$ shown to be necessary by the interaction-mating experiment (Fig. 2D). It was also noted that IB1 $1_{360-711}$ more preferentially bound to the cytoplasmic domain of APP than the cytoplasmic domains of APLP1 and APLP2, under the conditions that were used.
This IB1-APP interaction was reproduced by using its mouse counterpart, T7-JIP-1 $\mathrm{b}_{365-707}$, the homologous region encoded by HA-IB1 $1_{360-711}$. When the lysate of COS cells transfected with T7-JIP-1 $b_{365-707}$ was again precipitated with GST-fused proteins immobilized on glutathione beads, a weak but significant band corresponding to JIP-1 $\mathrm{b}_{365-707}$ was detected in the GST-cyt precipitant, but not in any of the other precipitants (Fig. 4B). The weakness of the JIP-1b immunoreactivity in the GST-cyt precipitant was attributed to proportionally weak expression of

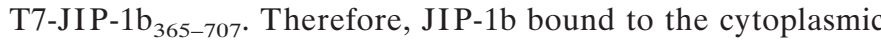
domain of APP in a manner similar to that observed for its human homolog IB1.

When T7-JIP-1 $365-660$, equivalent to JIP-1 b $_{365-707}$ lacking 47 amino acids in the PID region, was examined by the same GSTcyt pull-down experiment, no anti-T7-immunoreactive band was observed in the precipitants, although the construct was expressed sufficiently in the lysate (Fig. $4 B$ ). These results indicate that the PID region is essential for the JIP interaction with APP, the same observation obtained by the interaction-mating experiment shown in Figure 2D. The amounts of GST-fusion proteins contained in the precipitants were similar in all experiments above. The typical CBB staining is shown (Fig. $4 B, C B B$ ).

As negative controls, the lysate of mock-transfected cells was precipitated with immobilized GST-cyt, and their lysates and the ppts were subjected to immunoblotting using anti-HA and anti-T7 antibodies. Anti-HA antibody detected faint bands in the lysate, which are different in size from those found in the lysate of cells transfected with HA-IB1 ${ }_{360-711}$, but none in the precipitant. No immunoreactive bands were detected in either lysate or the precipitant with anti-T7 antibody (Fig. 4C). This confirms that the bands detected in the lysates or precipitants in Figure $4 B$ were derived from the transfected plasmids.

We were unable to perform interaction-mating assays using the bait fusions of the cytoplasmic domain of APLP1 and APLP2 because of their autoactivation of the LEU2 reporter gene (data not shown).

\section{GST-fused JIP-1b/IB1 can precipitate full-length APP with or without FAD mutations}

To further confirm the interaction of JIP-1b/IB1 with APP, reciprocal pull-down experiments were performed using GSTfused JIP-1b/IB1 constructs and full-length wt APP. When the lysates of COS cells transfected with wt APP and GST, GSTIB1 $_{360-711}$, GST-JIP-1b ${ }_{493-707}$, or GST-JIP-1b 540-707 , APP immunoreactivity was found in the glutathione-bead precipitants from lysates transfected with any GST-JIP-1b/IB1 construct but not in the precipitant from the lysate transfected with GST alone (Fig. 5A). Similar amounts of APP were detected in lysates of these transfected cells (Fig. 5A). Thus, GST-fused JIP-1b/IB1 constructs interacted with full-length wt APP.

We next examined whether this interaction was affected by FAD mutations in APP. For this purpose, COS cells were transfected with GST-IB1 $1_{360-711}$ and pEF-BOS, wt APP, or four FAD mutants of $\mathrm{APP}_{695}[\mathrm{~K} 595 \mathrm{~N} / \mathrm{M} 596 \mathrm{~L}-\mathrm{APP}$ (NL-APP), V642GAPP, V642F-APP, and V642I-APP] (Fig. 5B). The levels of expression of APP constructs were similar among wt APP and mutant APPs (Fig. 5B). When these lysates were precipitated with glutathione beads, APP immunoreactivity was detected in the precipitants from lysates transfected with either APP construct. This finding indicates that GST-IB1 $1_{360-711}$ can interact, to similar degrees, with full-length APP with or without FAD-associated mutations. Similar results were obtained by a pull-down experi- 

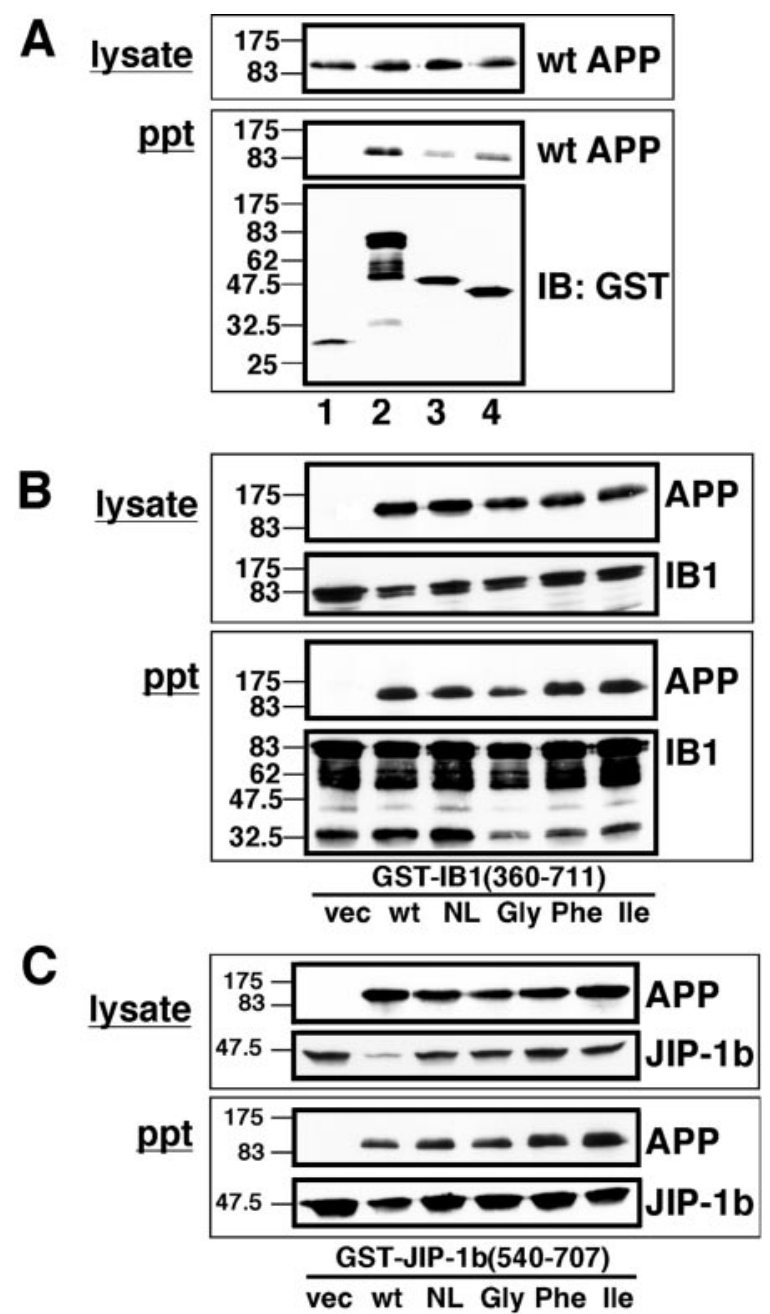

Figure 5. The C terminus of JIP-1b/IB1 precipitates wild-type and FAD mutant APPs. $A$, COS cells were cotransfected with wt APP with GST, GST-IB1 $1_{360-711}$, GST-JIP-1b ${ }_{493-707}$, or GST-JIP-1b ${ }_{540-707}$ (lanes 1-4, respectively) and precipitated with glutathione beads. The presence of APP and GST-fused proteins were probed with anti-APP antibody and antiGST antibody, respectively. The numbers on the left are the relative molecular weights of the size markers in kilodaltons. Each datum presented in Figure 5 is the representative of three independent experiments. $B$, COS cells were cotransfected with GST-IB1 $1_{360-711}$ with pEF-BOS (vec), wt APP (wt), NL-APP (NL), V642G-APP (Gly), V642F-APP (Phe), or V642I-APP (Ile) as indicated and precipitated with glutathione beads. Immunoreactivities of APP and GST-IB1 $1_{360-711}$ contained in the lysates (lysate) or precipitants ( $p p t$ ) were detected by anti-APP antibody and anti-GST antibody, respectively. $C$, Similar experiments were performed with COS cells transfected with GST-JIP-1b $b_{540-707}$ instead of GST-IB1 $1_{360-711}$. Other conditions and designations are the same as in $B$.

ment using GST-JIP-1b 540-707 instead of GST-IB1 $1_{360-711}$ (Fig. $5 C)$. JIP-1b/IB1 thus interacted similarly with wt APP and four FAD mutants (NL and V642I/F/G).

Anti-JIP-1b antisera precipitate full-length wt APP and APP mutants with JIP-1b expressed in COS cells and neuronal NT2 cells

When lysates of COS cells transfected with T7-JIP-1 $\mathrm{b}_{1-272}$ or T7-JIP-1 $b_{1-707}$ were probed with rabbit antiserum raised against the N-terminal residues $10-33$ of JIP-1b $(\alpha \mathrm{JIPN})$, the major immunoreactive bands at $\sim 40$ and $\sim 120 \mathrm{kDa}$ were those corresponding to the bands detected with anti-T7 antibody. In contrast, preimmune serum from the same rabbit failed to detect them (data not shown). Likewise, when lysates of cells transfected with T7-JIP-1 ${ }_{271-660}$ or T7-JIP-1 1-707 $_{1-1}$ were probed with rabbit antiserum raised against the $\mathrm{C}$-terminal residues $685-707$ of JIP-1b $(\alpha \mathrm{J}$ IPC), the major immunoreactive bands at $\sim 70$ and $\sim 120 \mathrm{kDa}$ were those corresponding to the bands detected with anti-T7 antibody, but its preimmune serum could not detect them (data not shown). This indicates that both $\alpha$ IIPN and $\alpha$ IPC antisera can specifically recognize transfected JIP-1b or its variants.

To further confirm the interaction of full-length JIP-1b with full-length APP, various combinations of plasmids were transfected into COS cells (Fig. 6A). When the lysates of transfected cells were analyzed by immunoblotting with anti-APP antibody, significant and similar amounts of wt APP and NL-APP were detected in the lysates of cells transfected with cognate constructs of APP, but little immunoreactivity of APP was detected in those transfected with the empty vector alone. Anti-T7 immunoreactivity of JIP-1b was detected in the lysates transfected with T7JIP-1b cDNA, but little immunoreactivity was detected in those transfected with an empty vector control (Fig. 6A). These lysates were immunoprecipitated with the antisera, as indicated in Figure $6 B$, using $\alpha \mathrm{JIPN}$ or its preimmune serum. The precipitants were subjected to immunoblot analysis using anti-APP and anti-T7 antibodies to detect wt APP or NL-APP and T7-JIP-1b, respectively. Significant immunoreactivity of JIP-1b was detected in the $\alpha$ JIPN precipitants from all of the T7-JIP-1b-transfected lysates, but no immunoreactivity was detected in those from the empty vector-transfected cells, nor was it detected in the precipitants by preimmune serum (Fig. $6 B$ ). This indicates that $\alpha$ JIPN antiserum precipitated JIP-1b in the presence or absence of exogenous APP expression. When the precipitants were probed using anti-APP antibody, APP immunoreactivity was detected in the $\alpha$ JIPN precipitants of cells transfected with both T7-JIP-1b and either wt APP or NL-APP, but not in the precipitant by preimmune serum. No APP immunoreactivity was detected when empty vector controls were used in place of either JIP-1b or APP (Fig. 6B). This indicates that the immunoprecipitation of JIP-1b was specific and that exogenous JIP-1b expression was required for APP to be precipitated by $\alpha \mathrm{JIPN}$ antiserum.

Essentially the same immunoprecipitation experiments were performed using $\alpha \mathrm{JIPC}$, yielding results similar to those obtained in the $\alpha$ JIPN experiments (Fig. 6C). Furthermore, similar results were obtained when similar immunoprecipitation was performed using V642I-APP instead of NL-APP (data not shown). Fulllength JIP-1b thus interacted with full-length APP with or without FAD mutations.

To further confirm the interaction of JIP-1b and APP in neuronal cells, wt APP and T7-JIP-1b or their corresponding empty vectors were transfected to NT2 cells, human neuronal precursor cells (Pleasure and Lee, 1993), as indicated in Figure $6 D$. When the lysates were subjected to immunoblotting and probed for the presence of APP and JIP-1b using anti-APP and T7-antibodies, respectively, APP and JIP-1b were detected in the lysates of cells transfected with their cognate plasmids. Significant amounts of APP of higher molecular weights were also detected in all lysates, including that of empty vector-transfected NT2 cells. These lysates were immunoprecipitated using $\alpha$ JIPC and its preimmune serum, and the precipitants were again probed for the presence of APP and JIP-1b. As in the experiments using COS cells, both JIP-1b and APP could be detected in the precipitant 
A

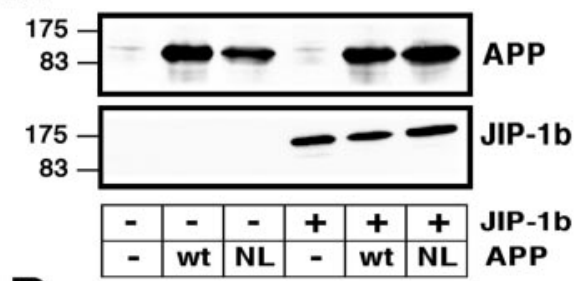

B

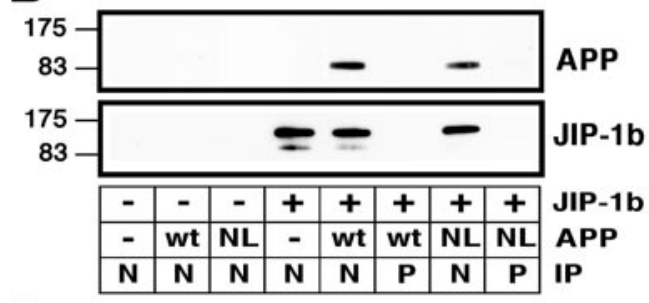

C

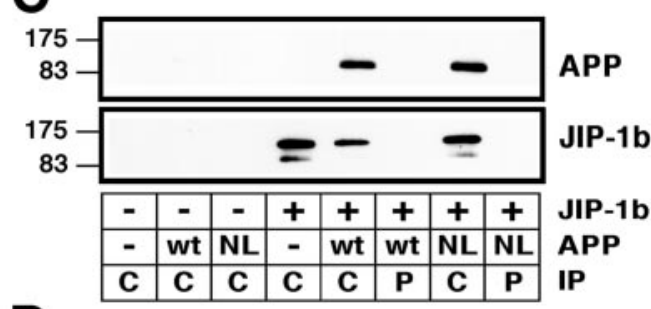

D
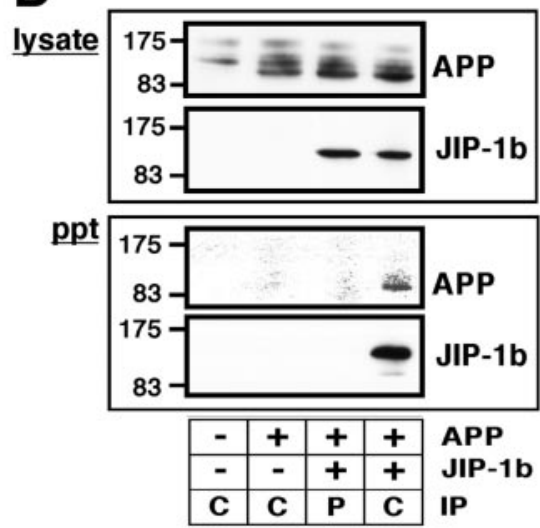

Figure 6. JIP-1b precipitates wild-type and FAD mutant APP in COS and NT2 cells. $A$, COS cells were transfected with the various combinations of wt APP, NL-APP, or their empty vector ( $w t, N L$, or - in APP, respectively), and T7-JIP-1b or its empty vector (+ or - in JIP-1b). The presence of APP and JIP-1b in the lysates was detected with anti-APP and anti-T7 antibody, respectively. The numbers on the left are the relative molecular weights of the size markers in kilodaltons. Each datum presented in Figure 6 is the representative of three independent experiments. $B$, The same lysates prepared from the transfectants of indicated plasmids were immunoprecipitated with the antisera $\alpha \mathrm{JIPN}(N)$ or its preimmune serum $(P)$. APP and JIP-1b were detected as in $A$. Other designations are the same as in $A$. $C$, Similar experiments were performed with $\alpha \mathrm{JIPC}(C)$ or its preimmune serum $(P)$. Other conditions and designations are the same as in $B . D$, Similar experiments were performed using neuronal NT2 cells transfected with the indicated combinations of wt APP and T7JIP-1b and $\alpha \mathrm{JIPC}(C)$ or its preimmune serum $(P)$. Their lysates (lysate) and precipitants $(p p t)$ were probed for the presence of APP and JIP-1b. Other conditions and designations are the same as in $C$.

from the lysate of NT2 cells transfected with both plasmids and precipitated with $\alpha \mathrm{JIPC}$, but not in the other combinations tested (Fig. 6D). The expression of JIP-1b in untransfected NT2 cells could not be detected using $\alpha$ JIPN or $\alpha$ JIPC (data not shown).

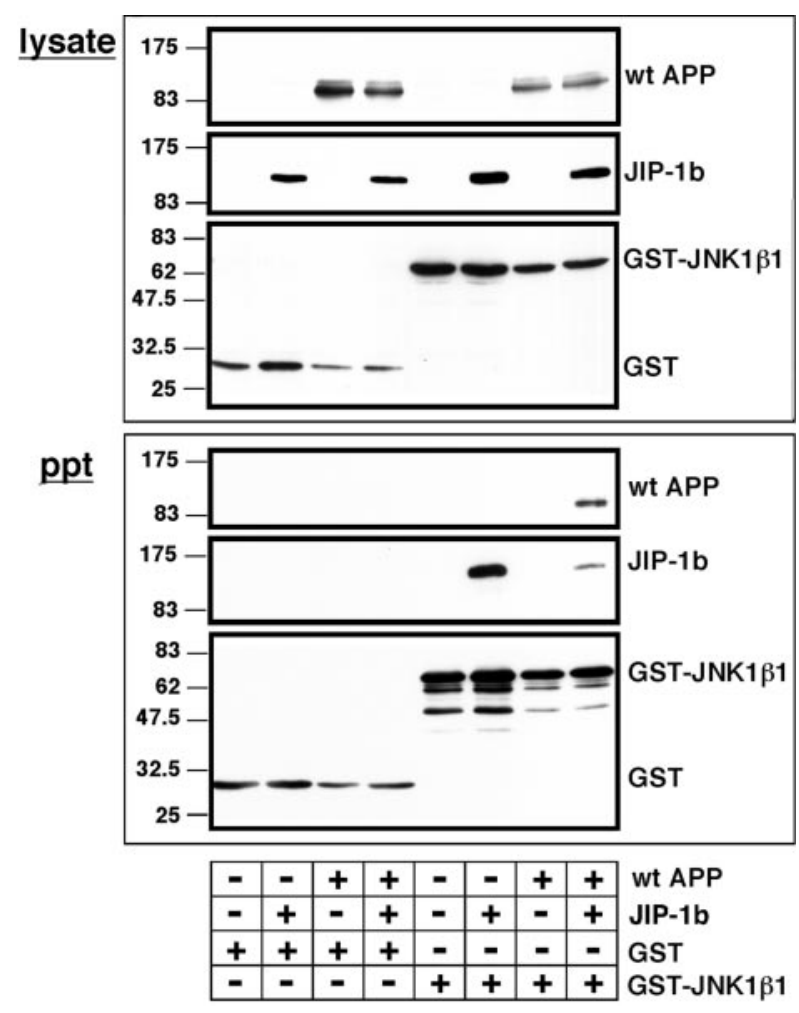

Figure 7. GST-fused JNK1 $\beta 1$ can precipitate APPs in a JIP-1bdependent manner. COS cells were transfected with the indicated combinations of wt APP or its empty vector ( + or - in $w t A P P)$, T7-JIP-1b or its empty vector (+ or - in JIP-1b), and GST or GST-JNK1ß1, as indicated with + under each lane, and their lysates were precipitated with glutathione beads. The presence of APP, JIP-1b, and GST-fused proteins in lysate (lysate) and precipitants (ppt) was detected with anti-APP, anti-T7, and anti-GST antibodies, respectively. Other designations are the same as in Figure 6. The data in this figure are representative of three independent experiments.

\section{GST-fused JNK1 $\beta 1$ precipitates wild-type and FAD mutant APP in the presence of exogenously expressed JIP-1b}

To investigate whether JIP-1b can scaffold JNK and APP, various combinations of plasmids were transfected to COS cells, and their lysates were probed with anti-APP, anti-T7, or anti-GST antibody to detect wt APP-, T7-JIP-1b-, and GST-fused proteins, respectively (Fig. 7, lysate). The protein expression of wt APP and JIP-1b was confirmed in the lysates of cells transfected with corresponding plasmids, but not in empty vector controls. Likewise, GST and GST-JNK1 $\beta 1$ were detected in cells transfected with the encoding plasmids, as the bands of the corresponding sizes of their expressed proteins (Fig. 7, lysate).

These lysates were precipitated with glutathione beads and subjected to immunoblot analyses (Fig. 7, ppt). GST or GSTJNK1 $\beta 1$ was detected in all precipitants from lysates transfected with the corresponding GST constructs. JIP-1b was detected in the precipitant from the lysate of cells transfected with both JIP-1b and GST-JNK1 $\beta 1$, but not in that from the lysate of cells transfected with GST alone nor the empty vector control of JIP-1b. This suggests that JIP-1b was specifically precipitated by JNK1b1. When the same precipitants were probed with anti-APP antibody, APP immunoreactivity was detected only when wt APP, JIP-1 $\beta$, and GST-JNK1 $\beta 1$ were cotransfected. In contrast, no APP immunoreactivity was detected in either precipitant from 


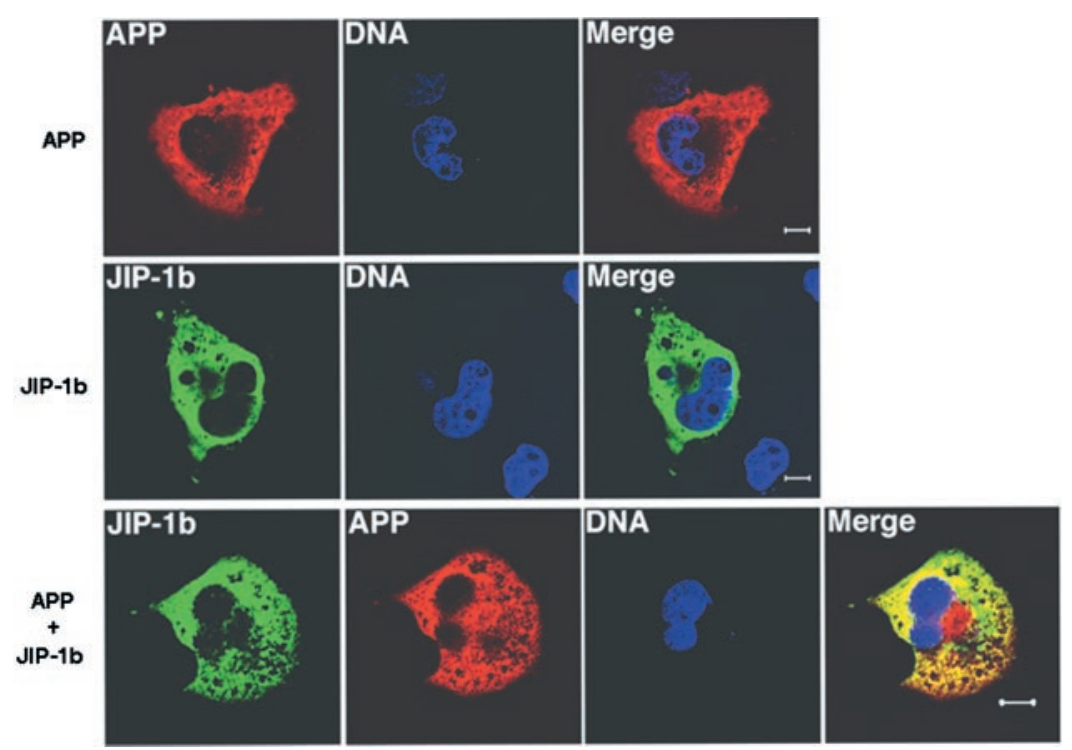

Figure 8. APP and JIP-1b share similar subcellular localization. COS cells were transfected with APP, JIP-1b, or both. JIP-1b expression was detected with $\alpha$ JIPN and visualized with FITC-conjugated secondary antibody (green). APP expression was detected with 22C11 (anti-APP antibody) and visualized with Texas Red-conjugated secondary antibody (red). DNA was visualized with Hoechst 33342 (blue), as described in Materials and Methods. The yellow area in the merged section shows where JIP-1b and APP share similar subcellular localization. Scale bars, $10 \mu \mathrm{m}$ The same set of experiments was repeated at least three times and yielded similar results. lysates transfected in the other combinations. This provides evidence that wt APP forms a complex with JNK1 $\beta 1$ through the scaffolding by JIP-1b.

\section{JIP-1b and APP share similar subcellular localization}

To further confirm the interaction of JIP-1b with APP in vivo, subcellular localization of JIP-1b and APP was examined by immunocytochemical analysis using confocal microscopy. When COS cells transfected with wt APP cDNA were stained with anti-APP antibody and visualized with Texas Red-conjugated secondary antibody, cells showed cytoplasmic staining but not nuclear staining (Fig. 8). No staining was observed when the same transfected cells were stained with control mouse IgG or when untransfected cells were stained with anti-APP antibody (data not shown).

When T7-JIP-1b-transfected cells were stained with $\alpha$ JIPN antiserum and visualized with FITC-conjugated secondary antibody, JIP-1b staining was observed in the cytoplasm and absent in the nucleus. Staining using $\alpha$ JIPC yielded similar results (data not shown). No staining was observed when untransfected cells were stained with $\alpha$ JIPN or $\alpha$ IIPC or when corresponding preimmune sera were used to stain T7-JIP-1b-transfected cells (data not shown).

To investigate whether JIP-1b and APP share subcellular localization, cells were cotransfected with T7-JIP-1b and APP and doubly stained by $\alpha$ JIPN and anti-APP antibody (Fig. 8). The immunostaining of JIP-1b (stained green) and that of APP (stained red) significantly overlapped in the shared subcellular localization, as revealed by the yellow area in the merged picture. These observations are consistent with the notion that a certain fraction of JIP-1b interacts with APP in vivo.

\section{DISCUSSION}

We have herein identified JIP-1b/IB1 as a novel APP-interacting molecule that indicated an affinity for the cytoplasmic domain of APP comparable to those of other known PID-containing APPinteracting proteins. JIP-1 was initially characterized as a cytoplasmic inhibitor of JNK family kinases (Dickens et al., 1997) and subsequently found to interact with MKK7, MLK, DLK, and HPK-1 in addition to JNK (Whitmarsh et al., 1998). Coexpression of JIP-1 and JNK with MKK7 or MLK3 increased JNK activation (Whitmarsh et al., 1998). These findings have estab- lished that JIP-1 scaffolds the kinase components of the JNK signaling pathway (Davis, 2000). An additional isoform of JIP-1 has been reported in mouse (JIP-1b) (Whitmarsh et al., 1998; Kim et al., 1999), rat [islet-brain-1 (IB1)] (Bonny et al., 1998), and human (IB1) (Mooser et al., 1999). This isoform contains a 47-residue insertion that completes the PID region at the $\mathrm{C}$ terminus, which was originally identified in Shc interaction with NPXY in the cytoplasmic domain of the epidermal growth factor receptor (Kavanaugh and Williams, 1994; Kavanaugh et al., 1995). Neither the physiological nor the pathological role of the JIP-1 proteins has become totally clear, whereas expression of JIP-1 has been reported to transcriptionally activate the GLUT2 promoter (Bonny et al., 1998) and is implicated in the pathogenesis of a form of type 2 familial diabetes mellitus (Waeber et al., 2000) and in the cytoprotection of insulin-secreting cells (Bonny et al., 2000). The present study thus provides the first line of evidence that the JNK scaffold protein, abundant in the brain and in islet $\beta$-cells, could be relevant to Alzheimer's disease. Interestingly, it has been reported that in vivo, neurotoxicity by hippocampal administration of Ab1-42 occurs only in diabetic rats (Smyth et al., 1994).

Analysis of subcellular localization using transfected cells indicates that JIP-1b and APP colocalized in the cytoplasm but both were not detected in the nuclei. Similar cytoplasmic localization of JIP-1b was reported by Dickens et al. (1997) and Whitmarsh et al. (1998), although Bonny et al. (1998) reported that IB1 is a nuclear protein. Coffey et al. (2000) showed nuclear localization of JIP-1 proteins in cerebellar granule cells, and Meyer et al. (1999) showed that JIP-1 proteins localize in the cytoplasm in unpolarized NIE115 and PC12 cells but are concentrated at neurites when the cells are polarized. These differences in JIP-1 localization thus may reflect different functions of JIP-1 proteins assigned in different cell environments. Although the present study provides evidence that JIP-1b interacts with APP inside the transfected cells, it would be necessary to investigate whether endogenous APP and JIP-1b interact in nontransfected cells. Yet the notion that JIP-1b/IB1 colocalizes with APP is consistent with earlier studies (Becker et al., 1999; Kim et al., 1999; Yasuda et al., 1999; Marcinkiewicz and Seidah, 2000; Pellet et al., 2000) indicating that the subcellular and brain regional localizations of JIP proteins considerably overlap with those of 
APP. Because the putative $\alpha$-secretase ADAM10 and the putative $\beta$-secretase BACE are expressed in the same neurons that express APP in the mouse brain (Marcinkiewicz and Seidah, 2000), APP cleavage by these putative secretases would lose the interaction of APP with JIP-1b/IB1, causing, in turn, a loss in the ability of JIP-1b/IB1 to specifically colocalize signaling molecules with APP. Although so far we have been unable to coimmunoprecipitate APP with IB1 from rat brain homogenates (data not shown), it remains unclear whether this failure is caused by inappropriate experimental conditions for specific immunoprecipitation of the APP/IB1 complex from solubilized brain homogenates or whether it implies that, with the APP/IB1 complex being a minor fraction, the majority of APP and IB1 in the brain does not complex with each other or form complexes with different partners. The latter notion is consistent, at least in part, with the observed relatively lower maximal binding of JIP-1b to the cytoplasmic domain of APP, as compared with those of the other PID-containing proteins tested.

By constructing deletion and point mutants, we have shown that the domains necessary for the APP/JIP-1b interaction are

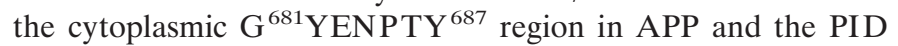
region in $\mathrm{JIP}-1 \mathrm{~b}$, completed by the insertion specific for this isoform. This accounts for the PID-nonbearing isoform JIP-1 not interacting with APP. As noted above, X11, Fe65, Fe65L, and mDab1 have been shown to interact with the $\mathrm{C}$ terminus of APP. The present study indicates that the APP/JIP-1b interaction requires $\mathrm{Tyr}^{682}, \mathrm{Asn}^{684}$, and $\mathrm{Tyr}^{687}$ contained in the $\mathrm{G}^{681} \mathrm{YENPTY}^{687}$ region. This is different from the mode of APP interaction with Fe65 and X11 and similar to that with mDab1. The observed amino acids required for the interaction of Fe65 or X11 concur with a previous report (Borg et al., 1996). Interestingly, the JIP-1b isoform, which is interactive with APP, is the major transcript in the brain, and the noninteractive JIP-1 transcript is hardly detected (Coffey et al., 2000; Pellet et al., 2000), pointing to certain specific roles of the JIP-1b isoform in neuronal functions.

The mechanism underlying the observed JIP-1b/IB1 interaction with APP is thus consistent with the established NPXY motif interaction of PID in Shc (Kavanaugh and Williams, 1994; Kavanaugh et al., 1995; Songyang et al., 1995) and IRS-1(Pawson and Scott, 1997). Yet in the present GST pull-down experiments, the cytoplasmic domains of APP, APLP1, and APLP2, all of which contain the same NPXY structure GYENPTY, showed largely different binding intensities for JIP-1b/IB1, with APP being the strongest among them. These different binding characteristics might reflect the difference in the primary to ternary structures surrounding the NPXY motif, suggesting the presence of an additional structural requirement allowing NPXY to interact efficiently with PID. In support of this idea, the most recent literature, in which PID of JIP-1b is shown to interact with p190 rhoGEF (Meyer et al., 1999), indicates that the binding region of p190 does not contain the classical NPXY motif.

Because JIP-1b showed binding similar to full-length APP regardless of the presence of four different FAD mutations, JIP-1b is most likely involved in the basic function of APP. Although the binding of Fe65 or X11 to APP has been shown to affect A $\beta$ secretion from APP (Borg et al., 1998; Sastre et al., 1998; Sabo et al., 1999; Tomita et al., 1999), so far we have not observed remarkable changes in A $\beta 42$ secretion from NL-APP by cotransfection with JIP-1b (Z. Shao, S. Matsuda, and I. Nishimoto, unpublished observation). The JIP-1 proteins have been shown to serve as scaffold proteins for the organization of active
JNK signaling complexes (Whitmarsh et al., 1998). In fact, we have shown in this study that APP associates with JNK via JIP-1b. It has also been established that APP interacts with the GTP-binding protein $\mathrm{G}_{\mathrm{o}}$ through the middle portion in the APP cytoplasmic domain adjacent to the NPXY-containing $\mathrm{C}$ terminus (Nishimoto et al., 1993; Okamoto et al., 1995; Brouillet et al., 1999). It is likely, therefore, that APP may serve as a membraneanchoring protein that further scaffolds the JIP-scaffolding complex with other signaling molecules. Taking into account the recently cloned members of the JIP family, JIP2 and JIP3 (Yasuda et al., 1999; Kelker et al., 2000)_PID is contained in JIP2 but not in JIP3 - it would deserve investigation whether APP might regulate the JNK signaling pathway through the binding of these various JIP proteins to the cytoplasmic domain of APP.

\section{REFERENCES}

Ausubel FM, Brent R, Kingston RE, Moore DD, Seidman JG, Smith JA, Struhl K (1999) Current protocols in molecular biology. New York: Wiley.

Becker AJ, Gillardon F, Blumcke I, Langendorfer D, Beck H, Wiestler OD (1999) Differential regulation of apoptosis-related genes in resistant and vulnerable subfields of rat epileptic hippocampus. Mol Brain Res 67:172-176.

Bonny C, Nicod P, Waever G (1998) IB1, a JIP-1-related nuclear protein present in insulin-secreting cells. J Biol Chem 273:1843-1846.

Bonny C, Oberson A, Steinmann M, Schorderet DF, Nicod P, Waeber G (2000) IB1 reduces cytokine-induced apoptosis of insulin-secreting cells J Biol Chem 275:16466-16472.

Borg J-P, Ooi J, Levy E, Margolis B (1996) The phosphotyrosine interaction domains of X11 and Fe65 bind to distinct sites on the YENPTY motif of amyloid precursor protein. Mol Cell Biol 16:6229-6241.

Borg J-P, Yang Y, De Taddeo-Borg M, Margolis B, Turner RS (1998) The X11 $\alpha$ protein slows cellular amyloid precursor protein processing and reduces $A \beta 40$ and $A \beta 42$ secretion. J Biol Chem 273:14761-14766.

Bork P, Margolis B (1995) A phosphotyrosine interaction domain. Cell 80:693-694.

Brouillet E, Trembleau A, Galanaud D, Volovitch M, Bouillot C, Valenza C, Prochiantz A, Allinquant B (1999) The amyloid precursor protein interacts with $G_{o}$ heterotrimeric protein within a cell compartment specialized in signal transduction. J Neurosci 19:1717-1727.

Coffey ET, Hongisto V, Dickens M, Davis RJ, Courtney MJ (2000) Dual roles for c-Jun N-terminal kinase in developmental and stress responses in cerebellar granule neurons. J Neurosci 20:7602-7613.

Coulson EJ, Barrett GL, Storey E, Bartlett PF, Beyreuther K, Masters CL (1997) Down-regulation of the amyloid protein precursor of Alzheimer's disease by antisense oligonucleotides reduces neuronal adhesion to specific substrata. Brain Res 770:72-80.

Davis RJ (2000) Signal transduction by the JNK group of MAP kinases. Cell 103:239-252.

Dickens M, Rogers JS, Cavanagh J, Raitano A, Xia Z, Halpern JR, Greenberg ME, Sawyers CL, Davis RJ (1997) A cytoplasmic inhibitor of the JNK signal transduction pathway. Science 277:693-696.

Duilio A, Faraonio R, Minopoli G, Zambrano N, Russo T (1998) Fe65L2: a new member of the Fe65 protein family interacting with the intracellular domain of the Alzheimer's $\beta$-amyloid precursor protein. Biochem J 330:513-519.

Finley Jr R, Brent R (1994) Interaction mating reveals binary and ternary connections between Drosophila cell cycle regulators. Proc Natl Acad Sci USA 91:12980-12984.

Fiore F, Zambrano N, Minopoli G, Donini V, Duilio A, Russo T (1995) The regions of the Fe65 protein homologous to the phosphotyrosine interaction/phosphotyrosine binding domain of Shc bind the intracellular domain of the Alzheimer's amyloid precursor protein. J Biol Chem 270:30853-30856.

Giambarella U, Yamatsuji T, Okamoto T, Matsui T, Ikezu T, Murayama Y, Levine MA, Katz A, Gautam N, Nishimoto I (1997) G protein $\beta \gamma$ complex mediated apoptosis by familial Alzheimer's disease mutant of APP. EMBO J 16:4897-4907.

Gietz RD, Schiestl RH (1995) Transforming yeast with DNA. Mol Cell Biol 5:255-269.

Gillian AM, McFarlane I, Lucy FM, Overly C, McConlogue L, Breen KC (1997) Individual isoforms of the amyloid $\beta$ precursor protein demonstrate differential adhesive potentials to constituents of the extracellular matrix. J Neurosci Res 49:154-160.

Gubler U, Hoffman BJ (1983) A simple and very efficient method for generating cDNA libraries. Gene 25:263-269.

Guenette SY, Chen J, Jondro PD, Tanzi RE (1996) Association of a novel human FE65-like protein with the cytoplasmic domain of the $\beta$-amyloid precursor protein. Proc Natl Acad Sci USA 93:10832-10837. 
Hardy J (1992) Framing $\beta$-amyloid. Nat Genet 1:233-234.

Hashimoto Y, Niikura T, Ito Y, Nishimoto I (2000) Multiple mechanisms underlie neurotoxicity by different types of Alzheimer's disease mutations of amyloid precursor protein. J Biol Chem 275:34541-34551.

Hirai S, Izawa M, Osada S, Spyrou G, Ohno S (1996) Activation of the JNK pathway by distantly related protein kinases, MEKK and MUK. Oncogene 12:641-650.

Jung SS, Nalbantoglu J, Cashman NR (1996) Alzheimer's $\beta$-amyloid precursor protein is expressed on the surface of immediately ex vivo brain cells: a flow cytometric study. J Neurosci Res 46:336-348.

Kang J, Lemaire H-G, Unterback A, Salbaum JM, Masters CL, Grezeschik KH, Multhaup G, Beyreuther K, Müller-Hill B (1987) The precursor of Alzheimer disease amyloid A4 protein resembles a cellsurface receptor. Nature 325:733-736.

Kavanaugh WM, Williams LT (1994) An alternative to SH2 domains for binding tyrosine-phosphorylated proteins. Science 266:1862-1865.

Kavanaugh WM, Turck CW, Williams LT (1995) PTB domain binding to signaling proteins through a sequence motif containing phosphotyrosine. Science 268:1177-1179.

Kelkar N, Gupta S, Dickens M, Davis RJ (2000) Interaction of a mitogen-activated protein kinase signaling module with the neuronal protein JIP3. Mol Cell Biol 20:1030-1043.

Kim IJ, Lee KW, Park BY, Lee JK, Park J, Choi IY, Eom SJ, Chang TS, Kim MJ, Yeom YI, Chang SK, Lee YD, Choi EJ, Han PL (1999) Molecular cloning of multiple splicing variants of JIP-1 preferentially expressed in brain. J Neurochem 72:1335-1343.

Lu DC, Rabizadeh S, Chandra S, Shayya RF, Ellerby LM, Ye X, Salvesen GS, Koo EH, Bredesen DE (2000) A second cytotoxic proteolytic peptide derived from amyloid $\beta$-protein precursor. Nat Med 6:397-404.

Luo JJ, Wallace W, Riccioni T, Ingram DK, Roth GS, Kusiak JW (1999) Death of PC12 cells and hippocampal neurons induced by adenoviralmediated FAD human amyloid precursor protein gene expression. J Neurosci Res 55:629-642.

Marcinkiewicz M, Seidah NG (2000) Coordinated expression of $\beta$-amyloid precursor protein and the putative $\beta$-secretase BACE and $\alpha$-secretase ADAM10 in mouse and human brain. J Neurochem 75:2133-2143.

Meroni G, Reymond A, Alcalay M, Borsani G, Tanigami A, Tonlorenzi R, Nigro CL, Messali S, Zollo M, Ledbetter DH, Brent R, Ballabio A, Carrozzo R (1997) Rox, a novel bHLHZip protein expressed in quiescent cells that heterodimerizes with Max, binds a non-canonical E box and acts as a transcriptional repressor. EMBO J 16:2892-2906.

Meyer D, Liu A, Margolis B (1999) Interaction of c-Jun amino-terminal kinase interacting protein-1 with p190 rhoGEF and its localization in differentiated neurons. J Biol Chem 274:35113-35118.

Mizushima S, Nagata S (1990) pEF-BOS, a powerful mammalian expression vector. Nucleic Acids Res 18:5322.

Mooser V, Maillard A, Bonny C, Steinmann M, Shaw P, Yarnall DP, Burns DK, Schorderet DF, Nicod P, Waeber G (1999) Genomic organization, fine-mapping, and expression of the human Islet-Brain 1 (IB1)/c-jun-amino-terminal kinase interacting protein-1 (JIP-1) gene. Genomics 55:202-208.

Murayama Y, Takeda S, Yonezawa K, Giambarella U, Nishimoto I, Ogata E (1996) Cell surface receptor function of amyloid precursor protein that activates Ser/Thr kinases. Gerontology 42[Suppl 1]:2-11.

Nishimoto I, Okamoto T, Matsuura Y, Takahashi S, Okamoto T, Murayama Y, Ogata E (1993) Alzheimer amyloid protein precursor complexes with brain GTP-binding protein $G_{0}$. Nature 362:75-79.

Okamoto T, Takeda S, Murayama Y, Ogata E, Nishimoto I (1995) Ligand dependent $\mathrm{G}$ protein coupling function of amyloid transmembrane precursor. J Biol Chem 270:4205-4208.

Okamoto T, Takeda S, Giambarella U, Matsuura Y, Katada T, Nishimoto I (1996) Intrinsic G-coupling function of amyloid precursor protein as a novel target of V642 mutations linked to familial Alzheimer disease. EMBO J 15:3769-3777.

Pawson T, Scott JD (1997) Signaling though scaffold, anchoring, and adaptor proteins. Science 278:2075-2080.

Pellet J-B, Haefliger J-A, Staple JK, Widmann C, Welker E, Hirling H,
Bonny C, Nicod P, Catsicas S, Waeber G, Riederer BM (2000) Spatial, temporal and subcellular localization of islet-brain 1 (IB1), a homologue of JIP-1, in mouse brain. Eur J Neurosci 12:621-632.

Perez RG, Zheng H, Van der Ploeg LHT, Koo EH (1997) The $\beta$-amyloid precursor protein of Alzheimer's disease enhances neuron viability and modulates neuronal polarity. J Neurosci 17:9407-9414.

Pleasure SJ, Lee VM (1993) NTera 2 cells: a human cell line which displays characteristics expected of a human committed neuronal progenitor cell. J Neurosci Res 35:585-602.

Qiu WQ, Ferreira A, Miller C, Koo E, Selkoe DJ (1995) Cell-surface $\beta$-amyloid precursor protein stimulates neurite outgrowth of hippocampal neurons in an isoform-dependent manner. J Neurosci 15:2157-2167.

Rohn TT, Ivins KJ, Bahr BA, Cotman CW, Cribbs DH (2000) A monoclonal antibody to amyloid precursor protein induces neuronal apoptosis. J Neurochem 74:2331-2342.

Sabo SL, Lanier LM, Ikin AF, Khorkova O, Sahasrabudhe S, Greengard P, Buxbaum JD (1999) Regulation of $\beta$-amyloid secretion by FE65, an amyloid protein precursor-binding protein. J Biol Chem 274:7952-7957.

Sanchez I, Hughes RT, Mayer B, Yee K, Woodgett JR, Avruch J, Kyriakis JM, Zon LI (1994) Role of SAPK/ERK kinase-1 in the stressactivated pathway regulating transcription factor c-Jun. Nature 372:794-798.

Sastre M, Turner RS, Levy E (1998) X11 interaction with $\beta$-amyloid precursor protein modulates its cellular stabilization and reduces amyloid $\beta$-protein secretion. J Biol Chem 273:22351-22357.

Smyth MD, Kesslak JP, Cummings BJ, Cotman CW (1994) Analysis of brain injury after intrahippocampal administration of $\beta$-amyloid in streptozotocin-treated rats. Neurobiol Aging 15:153-159.

Songyang Z, Margolis B, Chaudhuri M, Shoelson SE, Cantley LC (1995) The phosphotyrosine interaction domain of Shc recognizes tyrosine phosphorylated NPXY motif. J Biol Chem 270:14863-14866.

Sudo H, Jiang H, Yasukawa T, Hashimoto Y, Niikura T, Kawasumi M, Matsuda S, Takeuchi Y, Aiso S, Matsuoka M, Murayama Y, Nishimoto I (2000) Antibody-regulated neurotoxic function of cell surface $\beta$-amyloid precursor protein. Mol Cell Neurosci 16:708-723.

Tomita S, Ozaki T, Taru H, Oguchi S, Takeda S, Yagi Y, Sakiyama S, Kirino Y, Suzuki T (1999) Interaction of a neuron-specific protein containing PDZ domains with Alzheimer's amyloid precursor protein. J Biol Chem 274:2243-2254.

Trommsdorff M, Borg JP, Margolis B, Herz J (1998) Interaction of cytosolic adaptor proteins with neuronal apolipoprotein $\mathrm{E}$ receptors and the amyloid precursor protein. J Biol Chem 273:33556-33560.

Waeber G, Delplanque J, Bonny C, Mooser V, Steinmann M, Widmann C, Maillard A, Milklossy J, Dina C, Hani EH, Vionnet N, Nicod P, Boutin P, Froguel P (2000) The gene MAPK8IP1, encoding isletbrain-1, is a candidate for type 2 diabetes. Nat Genet 24:291-295.

Whitmarsh AJ, Cavanagh J, Tournier C, Yasuda J, Davis RJ (1998) A mammalian scaffold complex that selectively mediates MAP kinase activation. Science 281:1671-1674.

Wolozin B, Iwasaki K, Vito P, Ganjei JK, Lacana E, Sunderland T, Zhao B, Kusiak JW, Wasco W, D’Adamio L (1996) Participation of presenilin 2 in apoptosis: enhanced basal activity conferred by an Alzheimer mutation. Science 274:1710-1713.

Yamatsuji T, Okamoto T, Takeda S, Fukumoto H, Iwatsubo T, Suzuki N, Asami-Odaka A, Ireland S, Kinane TB, Nishimoto I (1996a) Neuronal DNA fragmentation by familial Alzheimer's V642 mutants of APP via heteromeric G proteins. Science 272:1349-1352.

Yamatsuji T, Okamoto T, Takeda S, Murayama Y, Tanaka N, Nishimoto I (1996b) Expression of V642 APP mutant causes cellular apoptosis as Alzheimer trait linked phenotype. EMBO J 15:498-509.

Yasuda J, Whitmarsh AJ, Cavanagh J, Sharma M, Davis RJ (1999) The JIP Group of mitogen-activated protein kinase scaffold proteins. Mol Cell Biol 19:7245-7254

Zhao B, Chrest FJ, Horton Jr WE, Sisodia SS, Kusiak JW (1997) Expression of mutant amyloid precursor proteins induces apoptosis in PC12 cells. J Neurosci Res 47:253-263. 